\title{
LA PERFORMATIVIDAD EN LA ACCIÓN PEDAGÓGICA DE PROFESORES/AS EN FORMACIÓN EN ARTES VISUALES*
}

\author{
THE PERFORMATIVITY IN THE PEDAGOGICAL ACTION \\ OF TEACHERS IN TRAINING IN VISUAL ARTS
}

\section{LUIS CLAUDIO CORTÉS P.**}

RESUMEN: La teoría de la performatividad contempla el cruce de numerosas disciplinas en torno a la tensa relación entre sanción y subversión a partir de la acción. La práctica profesional, en cuanto acción pedagógica de artes visuales en contextos escolares, contempla una serie de factores que sancionan y/o subvierten normas tendientes a conservar o transformar la realidad de niños/as y jóvenes a través del arte. A partir del proceso de supervisión de práctica profesional de ocho profesores/as de Artes Visuales en la Región Metropolitana, se analizan cuantitativa y cualitativamente sus reflexiones críticas, en torno a los siguientes factores: "cuerpo", "tiempo", "espacio", "materiales" y "tratamiento de contenidos". Se concluye que la acción pedagógica obedece a diversos hablas realizativos o performativos, acompañados de actos que transitan desde la sanción de normas imperantes en los contextos escolares, hacia la subversión de dichas normas.

Palabras Clave: Performatividad, Formación Inicial Docente, práctica profesional, artes visuales.

ABSTRACT: The theory of performativity encompasses numerous disciplines that converge around the tense relationship between sanction and subversion within any given action. The training experience of students in the field of visual arts pedagogy, as a form of pedagogical action within the school setting, involves a series of factors that sanction and/or subvert norms that aim to preserve or transform the reality of children and adolescents through art. Based on the process of supervising the teaching practicums of eight teachers in Santiago, Chile, this article offers a quantitative and qualitative analysis of their critical reflections on the following elements: body, time, space, materials, and the approach to content. We conclude that pedagogical action involves diverse actualizing and performative speech acts, which are accompanied by actions

* Artículo elaborado a partir del proyecto de investigación DIUMCE 2016, FIPEA 22-16 “Alcances Pedagógicos del Cuerpo y Performance en el Aula: Implementación de una Línea de Investigación en el Área de Prácticas Finales de Profesionales en Formación en Artes Visuales".

** Doctor en Educación Artística. Universidad Metropolitana de Ciencias de la Educación, Santiago, Chile. Correo electrónico: luis.cortes@umce.cl. 
that traverse the gamut from the sanction to subversion of prevailing norms within the school setting.

KeYwords: Performativity, initial teacher training, teaching practicums, visual arts.

Recibido: 14.06.17. Aceptado: 09.04.18.

\section{INTRODUCCIÓN}

R ajo el paradigma teórico-crítico (Adorno y Horkheimer, 2007), conB cretamente desde la pedagogía crítica (Mclaren, 1995; Giroux, 2003), la presente investigación emerge ante una problemática derivada de la práctica profesional de profesores/as de artes visuales en contextos escolares. Su principal cuestionamiento gira en torno a prácticas pedagógicas normadas por la creación de objetos y productos visuales (Acaso, 2009) obtenidos mediante procedimientos manuales, los cuales suponen aprendizaje y generan conocimiento a partir de criterios estético-formales (Acaso, 2013). El cuestionamiento emerge desde la teoría de la performatividad (Austin, 1990; Shechner, 2000; Taylor y Fuentes, 2011; Fischer-Lichte, 2013), en la medida en que todo "acto" deriva de "hablas" o "frases realizativas" que sancionan o subvierten normas imperantes al interior de las instituciones educativas, concretamente en el aula escolar (Mclaren, 1995).

$\mathrm{Su}$ objeto de estudio son las normas y sanciones culturales comprendidas como factores performativos tendientes al disciplinamiento de los lenguajes de creación visual derivados de las prácticas profesionales de 8 profesores/as en formación en artes visuales al interior de establecimientos educacionales en la Región Metropolitana. Dichos factores performativos son los siguientes: el disciplinamiento, la interdisciplinariedad, y la forma en que el cuerpo dinamiza su accionar de acuerdo a los materiales empleados, las técnicas y métodos manuales desarrollados, el tiempo y el espacio dispuesto para ello, y el tratamiento de los contenidos desarrollado por cada profesor/a en ejercicio al interior del aula, factores ampliamente analizados por Acaso, (2009), Llona (2011), Giráldez y Palacios (2014) y Errázuriz (2015).

Su objetivo principal es el siguiente: Comprender las acciones pedagógicas de profesores/as en artes visuales en formación, durante su práctica profesional, bajo la teoría de la performatividad, como marco de reflexión crítica ante la sanción y/o subversión de normas legitimadas culturalmente en el espacio escolar. 
Bajo estos términos se atiende la siguiente hipótesis en acción: La reflexión crítica en torno a sanciones y normas presentes en el accionar pedagógico de profesores/as de artes visuales durante su práctica profesional, permite comprender las posibilidades de transformación de dichas acciones bajo la teoría de la performatividad.

\section{MARCO TEÓRICO}

Tras la revisión de literatura especializada en torno a los estudios de performance (Shechner, 2000; Taylor y Fuentes, 2011; Fischer-Lichte, 2013) se arguye que el concepto "performance" deriva de la expresión en inglés "to perform", que significa "realizar", es decir, "realizar acciones" (Austin, 1990). La performance como proceso cultural interdisciplinar se percibe en el ámbito de las artes visuales (Barría, 2014), en los estudios lingüísticos (Austin, 1990), los estudios rituales (Hamui, 2011), los estudios teatrales (Fischer-Lichte, 2013), los estudios de género (Butler, 2005; Donaire, 2015) y de pedagogía crítica (Mclaren, 1995). Su objeto de estudio son los actos y acciones de los seres humanos en pleno desarrollo de su vida cotidiana al interior de instituciones que social y culturalmente se rigen por normas, las normas que son objeto de cuestionamiento a través de la acción misma, para efectos de ser subvertidas y dar curso a la transformación de la vida cotidiana.

En el plano lingüístico, Austin (1990) señala que desde la expresión verbal original en inglés "to perform", traducida como "realizar", se deriva a la expresión "performative" traducida como "realizativo". Ejemplo de ello son las siguientes expresiones verbales en primera persona del singular del presente de indicativo, en la voz activa: "te bautizo", "apuesto", "prometo", "te doy". Frases realizativas efectuadas conjuntamente por actos, "cuya realización es también la finalidad que persigue la expresión" (Austin, 1990, p. 49)

Ahora bien, dichas frases o expresiones verbales realizativas no tan solo se llevan a cabo con palabras, escritas o habladas, sino además se deben dar ciertas circunstancias. Para que el acto se lleve a cabo es necesaria la presencia de ciertas palabras, expresiones, personas o participantes, convenciones y procedimientos convencionales. Y puesto que son expresiones lingüísticas en las que decir algo es hacer algo no podrían calificarse de verdaderas o falsas, pero sí afortunadas o infortunadas, ya que el acto de llevarlas a cabo implica que en definitiva el acto se realiza (Austin, 1990). De hecho, Lyotard (1989), respecto del concepto de performatividad en el 
plano académico y empresarial, menciona que las acciones performativas corresponden a "juegos en los que la pertinencia no es ni la verdadera, ni la justa, ni la bella, etc., sino la eficiente" (p. 83).

De igual modo, Fischer-Lichte (2013) señala que la performatividad corresponde a realizaciones y/o acciones enunciadas que conforman actos sociales, puesto que "son actos lingüísticos autorreferenciales y constitutivos de realidad y como tales pueden tener éxito o fracaso, dependiendo sobre todo de condiciones institucionales y sociales" (p. 50).

Bajo estos términos, la performance conlleva procesos de performatividad derivados de acciones o actividades humanas inmersas en instituciones en las cuales confluyen la identificación de normas y la transgresión de las mismas (Mclaren, 1995).

En el contexto del aula, la performatividad es comprendida por Mclaren (1995) desde la pedagogía crítica como un proceso cultural normativo que gatilla subversión, toda vez que se modifican y desarticulan dinámicas culturales legitimadas institucionalmente. Se entiende por "normatividad" en el ámbito de las instituciones educativas a la sanción derivada de acciones o frases realizativas centradas en una educación basada en lecciones diarias, oraciones matutinas, ejercicios iniciales, asambleas escolares, etc. A su vez, con el término "subversión" se designan acciones derivadas de frases o actos realizativos generadores de resistencia a la instrucción, particularmente en las escuelas urbanas donde los rituales de la instrucción prevalecientes son a menudo cuestionados por grandes grupos de estudiantes, principalmente de clase trabajadora. Dichas acciones obedecen a performances ritualísticas que acarrean una fuerza material que pesa sobre los cuerpos y las mentes, tanto de maestros como de estudiantes. Para ello, Mclaren (1995) reflexiona críticamente cómo el poder está implicado en la sujeción del cuerpo a la cultura escolar, así como en el rechazo del cuerpo a renunciar a sus experiencias y deseos sedimentados.

De hecho, para Mclaren (1995), en el ritual y performance en la vida cotidiana de la escuela, "el concepto cuerpo es vuelto un símbolo maestro para el propósito de exégesis crítica sobre la escolarización como cultura del dolor. El dolor, sea existencial o físico, está íntimamente vinculado con la relación pedagógica" (p. 32).

En conclusión, la acción performativa al interior de las instituciones escolares sanciona y normativiza el cuerpo de los/las estudiantes y profesores/ as, sancionando ritualmente modos de estar y modos de ser de los cuerpos. En cambio, la subversión contempla su transformación, la transformación que no puede ser posible sino es a partir de la acción o acto realizativo 
subversivo, en donde profesores/as y estudiantes relacionados a partir del cuerpo al interior de dinámicas experimentales, suprimen la alteridad entre profesor/a y estudiante.

\section{METODOLOGÍA}

Investigación-Acción en el proceso de supervisión de práctica profesional

La presente investigación sigue el método de Investigación-Acción (Elliott, 2000). Su desarrollo se caracteriza por un proceso cíclico o un proceso de peldaños en espiral conformado por ciclos de investigación, en el que cada ciclo está formado por cuatro pasos fundamentales: planificación, acción, observación y reflexión.

Para atender dichos requerimientos y pasos metodológicos se define un ciclo de investigación por cada proceso de supervisión práctica, los cuales se conforman por tres pasos o peldaños: Reflexión previa a la acción/Observación de la acción/Reflexión de la acción.

En cada proceso de supervisión de práctica profesional, supervisor/a y practicante asumen un rol definido. El/la supervisor/a asume el rol de Investigador/a participante, mientras el/la practicante asume el rol de informante clave.

Se identifican además, dos espacios pedagógicos. El espacio de formación de profesores/as es identificado como $1^{\circ}$ espacio pedagógico y el espacio del aula en los centros de prácticas, como $2^{\circ}$ espacio pedagógico.

Por último, se definen dos técnicas de recogida de información: Observación participante y entrevista semiestructurada.

\section{Acceso al campo}

Acceder al campo de investigación contempló la supervisión de 8 practicantes. Cuatro durante el primer semestre y cuatro durante el segundo semestre de 2016. Estos practicantes fueron identificados con letra consecutiva del abecedario, con el objeto de mantener su anonimato.

Cada supervisión fue realizada bajo progresión cíclica, según método de Investigación-Acción, el cual contempló los siguientes pasos o peldaños:

1. Entrevista previa a la acción entre practicante y supervisor/a $\left(1^{\circ}\right.$ espacio pedagógico). 
2. Observación participante de la acción pedagógica de practicante en el aula ( $2^{\circ}$ escenario pedagógico).

3. Entrevista semiestructurada sobre la reflexión en torno a la acción de cada practicante ( $1^{\circ}$ espacio pedagógico) registrada en audio Mp3.

\section{Tratamiento de datos y resultados}

A raíz del proceso de recogida de información se obtienen 20 entrevistas semiestructuras registradas en audio $\mathrm{Mp} 3$. Dichas entrevistas se registran mediante proceso cíclico de supervisión, lo que permitió incrementar y robustecer las reflexiones críticas de cada acción práctica supervisada durante dos semestre académicos:

\begin{tabular}{|c|c|c|c|c|}
\hline $\mathrm{N}^{\circ}$ & $\begin{array}{l}\text { Practi- } \\
\text { cantes }\end{array}$ & $\begin{array}{l}\text { Número de } \\
\text { supervisiones }\end{array}$ & $\begin{array}{l}\text { Meses de registro entrevistas } \\
\text { semiestructuradas }\end{array}$ & $\begin{array}{l}\text { Semestres } \\
2016\end{array}$ \\
\hline \multirow{4}{*}{1} & \multirow{4}{*}{ A } & 1 & mayo & \multirow{11}{*}{$\begin{array}{c}1^{\mathrm{er}} \text { semestre } \\
2016\end{array}$} \\
\hline & & 2 & junio & \\
\hline & & 3 & junio & \\
\hline & & 4 & julio & \\
\hline \multirow{4}{*}{2} & \multirow{4}{*}{ B } & 1 & mayo & \\
\hline & & 2 & junio & \\
\hline & & 3 & junio & \\
\hline & & 4 & junio & \\
\hline \multirow{2}{*}{3} & \multirow{2}{*}{$\mathrm{C}$} & 1 & mayo & \\
\hline & & 2 & septiembre & \\
\hline 4 & $\mathrm{D}$ & 1 & agosto & \\
\hline \multirow{3}{*}{5} & \multirow{3}{*}{$\mathrm{E}$} & 1 & noviembre & \multirow{9}{*}{$\begin{array}{c}2^{\circ} \text { semestre } \\
2016\end{array}$} \\
\hline & & 2 & octubre & \\
\hline & & 3 & septiembre & \\
\hline \multirow{2}{*}{6} & \multirow{2}{*}{$\mathrm{F}$} & 1 & octubre & \\
\hline & & 2 & septiembre & \\
\hline \multirow{3}{*}{7} & \multirow{3}{*}{ G } & 1 & octubre & \\
\hline & & 2 & octubre & \\
\hline & & 3 & noviembre & \\
\hline 8 & $\mathrm{H}$ & 1 & noviembre & \\
\hline Total & 8 & & 20 & \\
\hline
\end{tabular}

Esta información posteriormente es transcrita y tratada en software NVIVO 11, que permite organizar la información cuantitativa y cualitativamente, mediante "nodos" y "referencias", comprendiendo por "nodos" cada 
categoría en estudio: cuerpo, espacio, tiempo, materiales y tratamiento de contenidos; y por "referencias", la alusión conceptual y semántica emanada de la reflexión crítica en torno a cada acción pedagógica, según practicante.

De este modo, los 8 practicantes entrevistados arrojan un total de 173 referencias, las que en definitiva corresponden a reflexiones críticas en torno a las cinco categorías en estudio, según características o naturaleza propia de cada acción pedagógica.

En la siguiente tabla es posible apreciar la distribución porcentual de referencias aludidas por los 8 practicantes a cada categoría en estudio:

\begin{tabular}{|l|c|c|c|c|c|c|c|c|c|c|}
\hline \multicolumn{1}{|c|}{ Practicantes } & A & B & C & D & E & F & G & H & \multicolumn{2}{c|}{ Total \% } \\
\hline Categorías & 0 & 9 & 2 & 7 & 1 & 10 & 8 & 2 & 39 & $23 \%$ \\
\hline Euerpo & 4 & 1 & 5 & 8 & 5 & 4 & 4 & 2 & 33 & $19 \%$ \\
\hline Tiempo & 1 & 5 & 7 & 6 & 3 & 2 & 2 & 4 & 30 & $17 \%$ \\
\hline Materiales & 4 & 9 & 6 & 7 & 2 & 5 & 8 & 3 & 44 & $25 \%$ \\
\hline $\begin{array}{l}\text { Tratamiento de } \\
\text { los contenidos }\end{array}$ & 4 & 5 & 4 & 3 & 2 & 6 & 3 & 0 & 27 & $16 \%$ \\
\hline Total & 13 & 29 & 24 & 31 & 13 & 27 & 25 & 11 & $\mathbf{1 7 3}$ & $100 \%$ \\
\hline
\end{tabular}

Cuerpo: La categoría "cuerpo" vislumbra las siguientes subcategorías, con sus respectivas referencias y relación porcentual:

\begin{tabular}{|l|l|c|c|}
\hline Categorías & Subcategorías & Referencias & $\%$ \\
\hline \multirow{4}{*}{ Cuerpo } & Desplazamiento del cuerpo en el espacio & 12 & $31 \%$ \\
\cline { 2 - 4 } & Primacía del cuerpo individual & 11 & $28 \%$ \\
\cline { 2 - 4 } & Relaciones físico-emocionales del cuerpo & 8 & $21 \%$ \\
\cline { 2 - 4 } & El cuerpo como soporte de comunicación & 8 & $20 \%$ \\
\hline
\end{tabular}

Desplazamiento del cuerpo en el espacio: Con un total de 12 referencias correspondientes al 31\% del total de la categoría cuerpo, se debe señalar que cada accionar pedagógico supervisado generó reflexiones respecto de los desplazamientos del cuerpo de practicantes y estudiantes en sus respectivos espacios de aprendizaje. Así por ejemplo, los estudiantes y practicantes transitaron entre espacios internos y externos a los centros de prácticas, lo que evidenció ventajas y desventajas en los desplazamientos de los cuerpos, según el tipo de espacio: sala de clases, taller, patio y espacio externo al centro de práctica. 
Primacía del cuerpo individual: Con un total de 11 referencias correspondientes a un $28 \%$ del total de la categoría cuerpo, se evidenció un predominio de actividades tendientes al trabajo individualizado en desmedro de actividades tendientes al desarrollo de relaciones corporales colectivas y colaborativas entre estudiantes y practicantes.

El cuerpo individualizado conserva directa relación con la creación de objetos artístico-visuales condicionados culturalmente por evaluación numérica:

La primera actividad que yo les hice, la hice sin nota. Ahora me pidieron nota. Y descubrí que la actividad no la toman en cuenta si no otorga un producto de esa naturaleza objetual, con el objetivo de que a ellos les sirva (B).

Relaciones físico-emocionales del cuerpo: Con un total de 8 referencias correspondientes al $21 \%$ del total de la categoría cuerpo, las relaciones físicoemocionales entre practicantes y estudiantes a partir del cuerpo generaron ambientes y climas óptimos de aprendizaje. Por ende, considerar el cuerpo como medio para alcanzar dicho fin, posiciona a el/la practicante ante el desafío de situarse en el lugar del otro, conocer a sus estudiantes en relación a sus necesidades físico-emocionales de aprendizaje.

El cuerpo como soporte de comunicación: Con un total de 8 referencias correspondientes al $20 \%$ del total de la categoría cuerpo, practicantes y estudiantes emplean sus cuerpos como soportes de comunicación, en la medida en que transmiten mensajes a partir de sus desplazamientos por el espacio del aula:

(...) el cuerpo es como tú te enfrentas al mundo, como tú te paras frente a los estudiantes, como ellos te perciben, es el mensaje. Es como cuando por ejemplo vemos una pintura de un action painting, lo que quizás estamos viendo son gotas de la huella que dejó el movimiento de la persona, entonces es un poco eso. Es como mi cuerpo va dejando esa huella, esa marca atrás. $(\mathrm{G})$

Espacio: Dentro de la categoría de "espacio" emergen las siguientes subcategorías con sus respectivas referencias y relación porcentual: 


\begin{tabular}{|c|c|c|c|c|}
\hline Categorías & & Subcategorías & Referencias & $\%$ \\
\hline \multirow{7}{*}{ Espacio } & \multicolumn{2}{|c|}{ Cantidad de estudiantes en Espacio } & 7 & $22 \%$ \\
\hline & \multirow{4}{*}{$\begin{array}{l}\text { Espacios } \\
\text { internos y } \\
\text { Espacios } \\
\text { externos }\end{array}$} & $\begin{array}{l}\text { Ventajas espacios internos: sala de } \\
\text { clases y talleres de artes }\end{array}$ & 7 & $21 \%$ \\
\hline & & $\begin{array}{l}\text { Desventajas espacios internos: Sala } \\
\text { de clases }\end{array}$ & 6 & $18 \%$ \\
\hline & & $\begin{array}{l}\text { Ventajas espacios internos: patio, } \\
\text { casino, cancha, jardines, accesos }\end{array}$ & 5 & $15 \%$ \\
\hline & & $\begin{array}{l}\text { Ventajas espacios externos: parque, } \\
\text { plaza, verdulería, taller, coloquio y } \\
\text { exposición }\end{array}$ & 5 & $15 \%$ \\
\hline & \multicolumn{2}{|c|}{$\begin{array}{l}\text { Supremacía Act. individuales v/s Act. } \\
\text { colaborativas }\end{array}$} & 2 & $6 \%$ \\
\hline & \multicolumn{2}{|c|}{ subvertir el espacio } & 1 & $3 \%$ \\
\hline
\end{tabular}

Cantidad de estudiantes en el espacio: Respecto de la cantidad de estudiantes en los espacios contemplados para las acciones prácticas, cabe señalar que un total de 7 referencias corresponden al $22 \%$ del total de la categoría espacio, lo que evidencia una clara preocupación ante la cantidad de estudiantes en el espacio destinado a las acciones prácticas, debido a lo cual emergen estrategias específicas para atender una cantidad numerosa de estudiantes, particularmente en el espacio de los talleres de artes.

Espacios internos y espacios externos: Respecto de los espacios internos y espacios externos, cabe señalar que se presentaron ventajas y desventajas para el desarrollo de las acciones prácticas.

En relación a espacios internos, tales como sala de clases y talleres de artes, con un total de 7 referencias correspondientes al 21\% del total de la categoría espacio, se presentaron ventajas respecto de las condiciones espaciales que garantizaban el desplazamiento del cuerpo, presentando condiciones adecuadas para el trabajo manual.

Particularmente, el espacio interno de la sala de clases, con un total de 6 referencias correspondiente al 18\% del total de la categoría espacio, presentan desventajas debido a lo reducido de su tamaño versus la cantidad de estudiantes en clase, lo que derivó en las limitaciones para el desplazamiento del cuerpo, condicionando el trabajo individualizado y ausencia de mobiliario adecuado. 
En cambio, los espacios internos al centro de práctica, tales como el patio, casino, paredes, cancha de fútbol, jardines y acceso al centro de práctica, con un total de 5 referencias correspondientes al 15\% del total de la categoría espacio, presentaron claras ventajas para las acciones pedagógicas, principalmente disruptivas. Estos espacios en donde se exhiben y exponen trabajos artístico-visuales elaborados por los y las estudiantes, también permiten el desarrollo de pinturas murales, acciones e instalaciones artísticas.

De igual modo, los espacios externos a los centros de práctica, tales como parque, plaza, verdulería, taller de artes, coloquio y exposición de artes, con un total de 5 referencias correspondientes al 15\% del total de la categoría espacio, presentaron claras ventajas al desarrollo de acciones pedagógicas performativas:

El tercero medio lo traje para el coloquio y allí funcionó bien porque los chicos son más tranquilos, pude conectar algunos contenidos que estábamos viendo y pasamos a ver la exposición de la Tirana en la sala Ana Cortés, entonces allí les expliqué un poco el proceso, pudieron verlos de cerca, ver el espacio, para poder comentarles que ellos también pueden exponer acá. (D)

Actividades individuales $v / s$ Actividades colaborativas: Con un total de 2 referencias correspondientes al $6 \%$ del total de la categoría espacio, se percibe de parte de un/a practicante la preferencia ante actividades individuales v/s actividades colaborativas, evidenciando, a su vez, una resistencia de parte de los y las estudiantes hacia el trabajo colectivo y colaborativo en talleres de artes de espacios reducidos.

Subvertir el espacio: Con un total de 1 referencia correspondiente al 3\% del total de la categoría espacio, un/a practicante promueve la subversión del espacio mediante modificación en la ubicación del mobiliario, así como además, prescindir de los materiales, con el objeto de deshabituar a los/las estudiantes. Lo anterior se debe a la carencia de comunicación entre pares, detectada por el/la practicante.

Tiempo: La categoría tiempo deriva en las siguientes subcategorías, referencias y relación porcentual: 


\begin{tabular}{|c|c|c|c|c|}
\hline Categoría & & Subcategorías & Referencias & $\%$ \\
\hline \multirow{7}{*}{ Tiempo } & \multirow{4}{*}{ Temporalidad } & Cierre & 9 & $27 \%$ \\
\hline & & Inicio & 7 & $23 \%$ \\
\hline & & Inicio, Desarrollo y Cierre & 3 & $10 \%$ \\
\hline & & Desarrollo & 1 & $3 \%$ \\
\hline & \multirow{3}{*}{$\begin{array}{l}\text { Concepciones } \\
\text { del tiempo }\end{array}$} & $\begin{array}{l}\text { Tiempo cronológico limitado } \\
90 \text { minutos }\end{array}$ & 7 & $23 \%$ \\
\hline & & $\begin{array}{l}\text { Tiempo destinado confección } \\
\text { objetos artístico-visuales }\end{array}$ & 2 & $7 \%$ \\
\hline & & $\begin{array}{l}\text { Tiempo aprendizaje } \\
\text { estudiantes }\end{array}$ & 2 & $7 \%$ \\
\hline
\end{tabular}

Temporalidad: La subcategoría temporalidad contempla cuatro componentes que dicen relación con la triada inicio/desarrollo/cierre. En primer lugar, el cierre con 9 referencias correspondientes al $27 \%$ del total de la categoría tiempo. En segundo lugar, el inicio con 7 referencias correspondientes al $23 \%$ del total de la categoría tiempo. En tercer lugar, la articulación inicio/desarrollo/cierre con 3 referencias correspondientes al 10\% del total de la categoría tiempo. Y, por último, el desarrollo con 1 referencia correspondiente al 3\% del total de la categoría tiempo.

Estos antecedentes evidencian que el tiempo y planificación previa de la acción práctica para dicho marco de tiempo dificultan cierres, inicios y articulación triádica, no así su desarrollo, por cuanto las acciones prácticas fundamentalmente destinan el tiempo a sesiones centradas en el hacer, ya sea manual o técnico, bajo procedimientos digitales, experienciales, salidas a terreno, etc.

Concepciones del tiempo: Respecto de las concepciones del tiempo que los y las practicantes poseen en torno a su acción práctica, se deben destacar tres aspectos:

El primero dice relación con lo limitado del tiempo destinado a las acciones prácticas, el cual contempla 90 minutos principalmente, cuyas 7 referencias corresponden al $23 \%$ del total de la categoría tiempo. El segundo, el tiempo destinado a la confección de objetos artístico-visuales, el cual contempla 2 referencias correspondientes al 7\% del total de la categoría tiempo. En tercer lugar, el tiempo considerado como tiempo de aprendizaje de los y las estudiantes, referencias que develan la preocupación por parte de los/las practicantes respecto del ritmo de aprendizaje de sus estudiantes, y cuyas 2 referencias corresponden al 7\% del total de la categoría tiempo. 
En este sentido, cabe destacar la siguiente referencia que alude a las limitaciones presentadas por los 90 minutos destinados a la asignatura de Artes Visuales:

Yo siento que 90 minutos es poquito, ojalá pudiesen ser dos días a la semana. Una cosa así. Quizás una clase de 90 y otra de 45 para que sirva, porque muchas veces la motivación se hace muy corta o durante la clase se trabaja súper bien, se avanzan bien los contenidos, los chicos viven bien el proceso de la experiencia que van teniendo, van identificando los contenidos en la medida que ellos producen. (D)

Materiales: A partir de la categoría materiales, los y las practicantes hacen referencias a las siguientes subcategorías, referencias y relación porcentual:

\begin{tabular}{|c|c|c|c|}
\hline Categoría & Subcategoría & Referencias & $\%$ \\
\hline \multirow{5}{*}{ Materiales } & Carencia de materiales en sesiones & 14 & $32 \%$ \\
\hline & $\begin{array}{l}\text { Dependencia uso materiales convencionales } \\
\text { conducentes objetos artístico-visuales }\end{array}$ & 14 & $32 \%$ \\
\hline & $\begin{array}{l}\text { Subversión dependencia y carencia acceso } \\
\text { materiales }\end{array}$ & 8 & $18 \%$ \\
\hline & $\begin{array}{l}\text { Materiales reciclaje y procedencia (abaratar } \\
\text { costos) }\end{array}$ & 7 & $16 \%$ \\
\hline & Materiales convencionales y materiales reciclaje & 1 & $2 \%$ \\
\hline
\end{tabular}

Carencia de materiales en sesiones: Con un total de 14 referencias correspondientes al $32 \%$ del total de la categoría materiales, se evidencia la carencia de materiales en las acciones prácticas planificadas y efectuadas. Al respecto, los y las practicantes señalan que carecen de materiales básicos y necesarios para un adecuado funcionamiento de su práctica profesional, poniendo énfasis que los y las estudiantes no llevan materiales, por lo cual son ellos/ellas quienes los suministran a sus estudiantes.

Dependencia uso materiales convencionales conducentes a objetos artístico-visuales: Con un total de 14 referencias correspondientes al 32\% del total de la categoría materiales, se evidencia una dependencia en el uso de materiales convencionales provenientes del mercado, conducentes a la elaboración de objetos artístico-visuales. Estos materiales conservan una relación de dependencia entre actividad y tipo de material, cuyo costo de 
acceso no se condice con las condiciones socioeconómicas de los y las estudiantes. Es decir, son materiales de alto costo, que entrampan el desarrollo de actividades propuestas por los/las practicantes. Debidos a esto, se emplean materiales de bajo costo con la finalidad de elaborar objetos artístico-visuales.

Subversión dependencia y carencia acceso materiales: Con un total de 8 referencias correspondientes al $18 \%$ del total de la categoría materiales, destacan acciones prácticas centradas en instalaciones artísticas con materiales efímeros, demandando el desplazamiento de estudiantes y practicantes en el espacio, sumado al factor sorpresa. De este modo se subvierte el espacio, la individualidad, la dependencia de los materiales y se pone énfasis en la experiencia, en lugar de adoptar contenidos transmitidos oralmente o elaborar objetos artístico-visuales, sustituyendo, en algunos casos, el material por el cuerpo como recurso didáctico.

Materiales reciclaje y procedencia (abaratar costos): Con un total de 7 referencias correspondientes al $16 \%$ del total de la categoría materiales, los y las practicantes diseñan estrategias alternativas para adquirir materiales, a partir de lo cual sus estudiantes sustituyen materiales convencionales por materiales reciclados:

Empecé desde lo que había, y luego cuando iba a tomar la micro empecé a ver que estaba lleno de vulcanización, había muchos bidones que se botaban, habían verdulerías cerca, entonces guié mi experiencia en torno a lo que había alrededor del colegio. Y la misma gente de alrededor del colegio me juntaba esos materiales y al final fueron a verlo como quedó. (C)

Materiales convencionales y materiales reciclaje: Con tan solo 1 referencia correspondiente al $2 \%$ del total de la categoría materiales, se reporta un caso que emplea, conjuntamente en sus acciones prácticas, materiales convencionales (provenientes del mercado) y materiales de reciclaje.

Tratamiento de los contenidos: Los y las practicantes atienden los contenidos específicos de su área disciplinar de Artes Visuales mediante las siguientes subcategorías, referencias y relación porcentual: 


\begin{tabular}{|c|c|c|c|}
\hline Categoría & Subcategorías & Referencias & $\%$ \\
\hline \multirow{4}{*}{$\begin{array}{c}\text { Tratamiento } \\
\text { de los } \\
\text { contenidos }\end{array}$} & $\begin{array}{l}\text { Contenidos y lenguajes de expresión visual } \\
\text { tratados }\end{array}$ & 16 & $59 \%$ \\
\hline & $\begin{array}{l}\text { Tratamiento de contenidos a partir de } \\
\text { necesidades del contexto }\end{array}$ & 5 & $19 \%$ \\
\hline & $\begin{array}{l}\text { Zona de confort y dominio específico de } \\
\text { lenguajes expresión visual }\end{array}$ & 3 & $11 \%$ \\
\hline & $\begin{array}{l}\text { Formación específica pregrado lenguaje } \\
\text { expresión visual }\end{array}$ & 3 & $11 \%$ \\
\hline
\end{tabular}

Contenidos y lenguajes de expresión visual tratados: $\mathrm{Al}$ respecto, se registran 16 referencias, las cuales corresponden al 59\% del total de la categoría "tratamiento del contenido". Dicha subcategoría configura tres grupos. El primero dice relación con el desarrollo de un lenguaje visual y un contenido específico. El segundo, con el desarrollo teórico de un contenido específico. Y el tercero, la relación interdisciplinar entre el discurso teórico y la creación de obra visual.

Tratamiento de contenidos a partir de necesidades del contexto: Con un total de 5 referencias, las cuales corresponden a un 19\% del total de la categoría "tratamiento del contenido", los y las practicantes atienden dichos contenidos teóricos y prácticos según las necesidades del contexto educativo. Así por ejemplo, algunos practicantes efectúan tratamiento de los contenidos considerando los niveles de aprendizaje de sus estudiantes, considerando además el tipo de centro educativo, ajustándose a los requerimientos institucionales, según demandas de establecimientos educacionales Científico Humanistas, Técnico profesionales o Experimental Artístico.

Zona de confort. Dominio específico de lenguajes de expresión visual: Con 3 referencias, correspondientes al 13\% del total de la categoría "tratamiento del contenido", los/las practicantes evidenciaron un dominio específico de su lenguaje visual. Este dominio se vio reflejado en su productividad de obra, lo cual posibilitó el tránsito conjunto con sus estudiantes desde una zona de confort disciplinar hacia su profundización mediante la experimentación.

Formación específica del pregrado en el desarrollo de un lenguaje de expresión visual: Con 3 referencias, correspondientes al 11\% del total de la categoría "tratamiento del contenido", los y las practicantes evidenciaron el desarrollo de un lenguaje de expresión visual particular de carácter subjetivo, cuya 
práctica develó un desarrollo disciplinar específico en los y las estudiantes a su cargo:

Al menos desde mi trayectoria o lo que he hecho durante la carrera en términos de propuesta visual, yo creo que sí se puede unir y puedo recurrir bastante a mi obra. Al menos mostrárselas a los chicos, comentar sobre eso. También buscar una especie de método para que ellos puedan desarrollarse de la misma forma que yo lo hice, desde el ámbito escolar quizás. Que ellos sintieran que podían proponer, al menos como lo sentí en mi formación de la carrera. (D)

\section{CONCLUSIONES}

A modo de conclusión, es posible señalar que evidentemente predominan acciones pedagógicas que sancionan normas imperantes, tales como la creación de objetos artístico-visuales, mediante procedimientos técnicos y manuales, principalmente de elaboración individual. Dichas acciones pedagógicas están condicionadas por el tiempo curricular destinado a la asignatura de Artes Visuales, así como también por las condiciones espaciales relacionadas con la cantidad de estudiantes en el interior de las salas. Estos son aspectos que en definitiva impiden atender una transformación de prácticas normadas y sancionadas de artes visuales en contextos escolares, en los términos señalados por Acaso (2009).

De este modo, comprender la práctica profesional de profesores/as en formación en artes visuales, bajo la teoría de la performatividad, permite recoger las sanciones que han de ser consideradas en el momento de innovar o transgredir normas que limiten el aprendizaje de las Artes Visuales.

De igual modo, pero en menor cantidad, las acciones pedagógicas tendientes a la subversión de dichas condiciones develaron estrategias en donde el cuerpo es crucial, sustituyendo los materiales convencionales por experiencias o instalaciones con materiales efímeros, realizando actividades fuera de la sala de clases y de los centros de práctica, en donde el espacio, el tiempo y los materiales se subvierten a partir de actos y hablas realizativos centrados en la experiencia, en lugar del resultado objetual. Por ende, el acto performativo que subvierte las normas de prácticas legitimadas por la elaboración de objetos y artefactos visuales configura un nuevo rol para el/ la profesor/a de Artes Visuales, que se cruza con otras disciplinas.

Por último, debemos señalar que dentro de las posibles aplicaciones prácticas de la presente investigación, destaca el impacto que esta podría 
tener al interior de los procesos de formación práctica de planes de formación inicial de docentes. Esta aplicación puede ser monitoreada en procesos de supervisión de prácticas tanto profesionales como progresivas, en la medida en que las categorías "cuerpo", "espacio", "tiempo", "materiales" y "tratamiento de los contenidos" se comportan como factores transversales indistintamente de la disciplina.

\section{REFERENCIAS}

Acaso, M. (2009). La educación artística no son manualidades. Nuevas prácticas en la enseñanza de las artes y la cultura visual. Madrid: Catarata.

Acaso, M. (2013). rEDUvolution: hacer la revolución en la educación. Barcelona: Paidós.

Adorno, T. y Horkheimer, M. (2007). Dialéctica de la Ilustración. Madrid: Akal. Austin, J. L. (1990). Cómo hacer cosas con palabras. Barcelona: Paidós Studio.

Barría, M. (2014). Intermitencias. Ensayos sobre performance, teatro y visualidad. Santiago de Chile: Editorial Universitaria.

Butler, J. (2005). Cuerpos que importan. Sobre los límites materiales y discursivos del sexo. Buenos Aires: Paidós.

Donaire, A. (2015). Pink Flamingos y lo abyecto: Entre performatividad paródica y pastiche. Atenea, 511, 175-187.

Elliott, J. (2000). El cambio educativo desde la investigación-acción. Madrid: Morata.

Errázuriz, L. H. (2015). El (f)actor invisible. Estética cotidiana y cultura visual en espacios escolares. Santiago de Chile: Consejo Nacional de la Cultura y las Artes.

Fischer-Lichte, E. (2013). Estética de lo performativo. Madrid: ABADA Editores.

Giráldez, A., y Palacios, A. (2014). Educación artística en Iberoamérica: Educación Primaria. Madrid: Organización de Estados Iberoamericanos, OEI.

Giroux, H.A. (2003). Pedagogía y política de la esperanza. Teoría, cultura y enseñanza. Buenos Aires: Amorrortu.

Hamui, S. (2011). El ritual como performance. Enunciación, 16(1), 16-30.

Llona, E. (2011). Estudio sobre el estado actual de la educación artística en la Región Metropolitana. Recuperado de http://www.idea-educa.cl/descargas/educacion_artistica.pdf

Lyotard, J. F. (1989). La condición posmoderna. Cátedra: Madrid.

Mclaren, P. (1995). La escuela como performance ritual. Hacia una economía política de los símbolos y gestos educativos. Siglo XXI: México.

Shechner, R. (2000). Peformance: Teoría y prácticas interculturales. Buenos Aires: Libros de Rojas UBA. 
Taylor, D. y Fuentes, M. (2011). Estudios avanzados de performance. México: Fondo de Cultura Económica. 